\title{
Influence of Inorganic Salts on Soils Liquid and Plastic Limits
}

\author{
Ayininuola, G.M. ${ }^{1}$ and Agbede, O.A. ${ }^{1}$
}

\begin{abstract}
This study focused on the influence of inorganic salts on soil liquid limit (LL) and plastic limit (PL). Sodium chloride, $\mathrm{NaCl}$, potassium nitrate $\mathrm{KNO}_{3}$ and calcium sulphate, $\mathrm{CaSO}_{4}$ of various concentrations were added to two subsoils. Their liquid and plastic limits before and after contamination were monitored. Soil cation exchange capacity (CEC), exchangeable cations (sodium, $\mathrm{Na}^{+}$, potassium, $\mathrm{K}^{+}$, and calcium, $\mathrm{Ca}^{2+}$ ) and exchangeable anions (chloride, $\mathrm{Cl}$, nitrate, $\mathrm{NO}_{3}$, and sulphate, $\mathrm{SO}_{4}{ }^{2}$ ) were determined. A set of equations for predicting $\mathrm{LL}$ and $\mathrm{PL}$, (with $\mathrm{R}^{2}$ $=0.908$ to 0.990 ) at different levels of chemical interaction with time was developed using multiple regression analysis model. The results showed that both $\mathrm{NaCl}$ and $\mathrm{KNO}_{3}$ brought about reduction in $\mathrm{LL}$ and $\mathrm{PL}$ while $\mathrm{CaSO}_{4}$ addition led to increment in $\mathrm{LL}$ and PL. The predictive equations revealed that there is high tendency for the contaminated soils to regain their uncontaminated LL and PL values with time.
\end{abstract}

Keywords: Cation exchange capacity, contamination, exchangeable cation, liquid limit, plastic limit, subsoils.

\section{Introduction}

Depending on water content, four stages of consistency exist in a soil: liquid, plastic, semi-solid and solid states. In wet condition, fine-grained soil acts like a viscous liquid and as it begins to dry, it acts like plastic. With further reduction in water content, the soil becomes brittle and subsequent loss of water makes it solid. Out of four limits that are in existence, only liquid and plastic limits retained their popularity [1]. Plastic consistency is used as a basis for fine grained soil classification. Foundation soils, homogeneous and heterogeneous in nature are coarse grained soil and fine grained soil. Coarse grained soils have no plastic state of consistency.

The presence of clay in fine grained soil and heterogeneous soils is responsible for their consistency due to its extremely flaky shape. Clay mineral has large surface area and adsorbed layers. In the adsorbed layer is adsorbed water. The total negative charges carried by all clay minerals are neutralized in different ways: by internal cations, hydrogen bonds, and cation in the adsorbed layer. The excess unbalanced negative charges are termed cation exchange capacities (CEC) that are neutralized by external cations from soil contaminants.

${ }^{1}$ Department of Civil Engineering, University of Ibadan, Ibadan, NIGERIA

*Corresponding author, e-mail: gm.ayininuola@mail.ui.edu.ng or ayigbenga@gmail.com

Note: Discussion is expected before June, $1^{\text {st }} 2013$, and will be published in the "Civil Engineering Dimension" volume 15, number 2, September 2013.

Received 9 November 2011; revised 25 May 2012; accepted 29 August 2012
The presence of certain cations tends to either increase or decrease the thickness of adsorbed layer. The process would have adverse effect on the soil consistency, which is the central focus of this study.

The original constituents of soil (solid, water, and air) are changing daily due to human activities. Indiscriminate dumping of wastes (liquid and refuse) is becoming rampart. The consequence of environmental pollution can be disastrous [2]. In studies carried out at Osogbo, Nigeria, the levels of lead, copper, calcium, nickel, and zinc at the motor parks and mechanic workshops were far above the levels at the normal sites [3]. A study carried out at coal fired power plant in Western Turkey showed that sodium, potassium, calcium, magnesium, manganese, aluminum, and iron contents of soil and water are governed by basement lithology, whereas lead, zinc, and copper contents are determined by fly ash from disposal sites [4]. Industrialisation and urbanisation are two main causes for increasing contamination of metals in soils [5].

Studies carried out by researchers have shown that on exposure to concentrated salt solutions, volume change of saturated clay soils occurred due to fluid flowing out of the clay, in response to chemical concentration gradients [6,7]. Using four different clay soils and three chlorides salts (sodium chloride, potassium chloride, and calcium chloride), decaying in the liquid limit of clay soils as salt molarity increased was observed [8]. The liquid limit of sodium-saturated sample of Bangkok-clay increased with increase in sodium chloride concentration. Substituting calcium for sodium in Ariake clay gives similar result, however the extent of the liquid limit change by such treatment was much smaller for Bangkok clay than Ariake clay [9]. 
The liquid limit of the sodium saturated Ariake clay decreased as the salinity of the pore water decreased [10]. The consistency limits of $\mathrm{Ca}$ and Na-bentonite with different concentrations of $\mathrm{CaCl}_{2}$ (varying between 0.01 and $0.735 \mathrm{M}$ ), $\mathrm{NaCl}$ (varying between 0.01 and $0.1 \mathrm{M}$ ), and methanol (pure methanol and $50 \%$ methanol in distilled water), and gasoline have been investigated. When mixed with a strong $\mathrm{CaCl}_{2}$ solution, calcium and sodium bentonite had approximately the same liquid limit and plasticity index [11]. The effect of $\mathrm{CaCl}_{2}$ solutions with concentration of $5,10,20,50,100$, and $500 \mathrm{mM}$ on the liquid limits of geosynthetic material that incorporates bentonite clay of low hydraulic conductivity used for lining landfill called Geosynthetic Clay Liners (GCLs) were carried out [12].

Furthermore, the effects of surfactants (octylphenol polyoxyethylene, biosurfactant, and sodium dodecyl sulfate) and electrolyte solutions $\left(\mathrm{NaPO}_{3}\right.$ and $\left.\mathrm{CaCl}_{2}\right)$ on some properties of two soil samples (100\% kaolinite clays soil and $30 \%$ kaolinite $+70 \%$ sand) were investigated. The result revealed that $\mathrm{CaCl}_{2}$ solution did not affect the liquid limit significantly but led to decrease in plasticity index of kaolinite soil [13].

The effect of brine on clay was conducted. The result showed that an increase in salt pore fluid concentration resulted in a decrease in the liquid limit, little or slight increase in the plastic limit [14]. Salt solution was used in-place of distilled water for determination of clay soils liquid and plastic limits. The results showed that increase in salts (copper sulphate, iron sulphate, and potassium chloride) concentration led to increase in CL-clay liquid and plastic limits. The reverse was obtained for $\mathrm{CH}$-clay [15]. In all of the literature examined, salts were added to water used in carrying out liquid and plastic limits experiments. The effect of actual reaction of salts with soil elements (minerals) over time was not considered. This salient issue and many others are addressed in this report.

\section{Materials and Methods}

Two subsoils were obtained from two burrow pits in Ibadan, Nigeria referred to as subsoils A and B. At the sampling points, the top soils were removed before enough quantities of subsoils were taken. The subsoil was mixed for homogeneity, sun-dried and stockpiled in labeled sacks for identification. Small portion of subsoil was set aside for the control experiments and the remaining was divided into sixty parts on flat platform. One hundred and twenty plastic containers of 120 liters capacity each were procured. In order to aid free flow of salts solution and elimination of excess salt accumulation, the bottom of each container was perforated with drilling machine. Each part of subsoil was poured into 120 liters container. Altogether, 180kg of soil was used to fill each container. About 20 liters overboard was left at the top of each container to be occupied by salt solution. The containers were labeled ASC-10, ASC-30, ASC-50, ASC-70, APN-10, APN-30, APN-50, APN-70, ACS-10, ACS-30, ACS50, ACS-70, BSC-10, BSC-30, BSC-50, BSC-70, BPN-10, BPN-30, BPN-50, BPN-70, BCS-10, BCS30, BCS-50, and BCS-70. Containers labeled SC, $\mathrm{PN}$, and $\mathrm{CS}$ received $\mathrm{NaCl}, \mathrm{KNO}_{3}$, and $\mathrm{CaSO}_{4}$ respectively, while the first alphabet stands for subsoil A and B and the last two digits on the labels stand for dosages in $\mathrm{g} / \mathrm{dm}^{3}$ of the salt solutions.

Enough quantities of the three procured salts (sodium chloride, $\mathrm{NaCl}$, potassium nitrate, $\mathrm{KNO}_{3}$ and calcium sulphate, $\mathrm{CaSO}_{4}$ ) were obtained. Standard solutions of each salt of concentrations 10, 30,50 , and $70 \mathrm{~g} / \mathrm{dm}^{3}$ were prepared. Each soil in plastic container received $18 \mathrm{dm}^{3}$ of single salt solution. The salt solutions were applied to the soils in containers by gravity. Excess salt solutions were seen on the floor gushing out from bottom of all containers within three hours of salt solutions application. This development showed that soil grains were fully embedded in the salt solution before commencement of laboratory tests. The 120 plastic containers were covered and placed in a shaded place throughout the duration of study as shown in Figure 1.

In line with British Standard [16], grain size analyses of the two subsoils were carried out and the liquid and plastic limits were determined. These two subsoils were taken as control soils. The liquid and plastic limits of soils contaminated with salt solutions were determined on day $7,14,28,56,112$, 196, 280, and 364, adopting the procedures for the controlled subsoils. Prior to each test, suitable quantity of contaminated soil taken from each container was air-dried to aid sieving operation. The chemical properties of both controlled subsoils and contaminated soils were determined. Since the soil was mixed together and only one salt solution was introduced to each soil in the container, only those ions present in the salt added were expected to vary significantly in each soil.

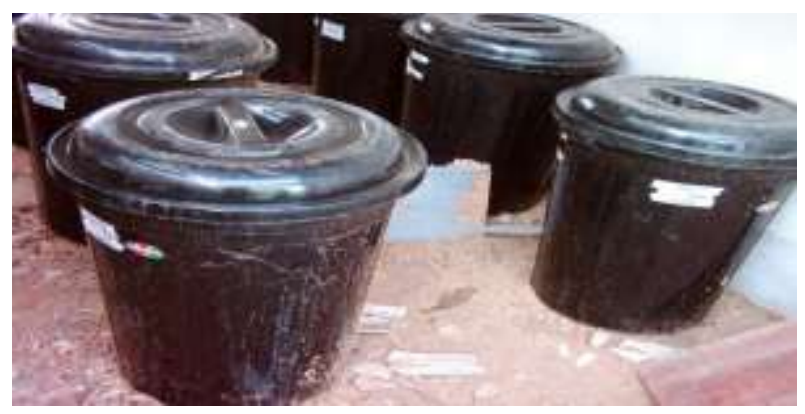

Figure 1. Contaminated Soils in a Shed 
Soils ASC-10 to ASC-70 and BSC-10 to BSC-70 exchangeable sodium $\mathrm{Na}^{+}$and chloride $\mathrm{Cl}$ - levels were monitored by atomic-emission spectrometer and colorimeter methods respectively.

Exchangeable potassium, $\mathrm{K}^{+}$, and nitrate, $\mathrm{NO}_{3}$ - levels present in soils APN-10 to APN-70 and BPN-10 to BPN-70 were determined with the aid of atomicemission spectrometer and nitrate electrode methods respectively. Also, exchangeable calcium $\mathrm{Ca}^{2+}$ and sulphate $\mathrm{SO}_{4}{ }^{2-}$ levels in soils ACS-10 to ACS-70 and BCS-10 to BCS-70 were determined by atomicemission spectrometer and nephelometer analysis methods respectively. In addition, the cation exchange capacity of controlled subsoils and contaminated soils were measured.

The field work revealed that the quantity of soil taken from each container for geotechnical properties determination with time have different values of CEC, exchangeable cations and anions. The revelation made it difficult to ascertain the response of soil geotechnical properties at constant level of CEC and exchangeable cations and anions with time. In order to investigate the variation in geotechnical properties of the subsoil with uniform chemical properties, a predictive model yielding a set of equations was developed. The model was developed for the two subsoils under the influence of the two salts separately.

Multiple linear regressions were used to model the relationship between the subsoil geotechnical properties (dependent variables) and chemical properties (independent variables) [17]. The general regression model is expressed by an equation

$\mu=B_{0}+b_{1} \mathrm{x}_{1}+B_{2} \mathrm{x}_{2}+B_{3} \mathrm{x}_{3}+\ldots \ldots \ldots \ldots .+B_{\mathrm{n}} \mathrm{x}_{\mathrm{n}}$

Where:

$\mu=$ Dependent variable

$\mathrm{x} 1, \mathrm{x}_{2}, \ldots \mathrm{x}_{\mathrm{n}}=$ Independent variables of order $\mathrm{n}$

$B_{1}, B_{2}, B_{3}, \ldots, B_{\mathrm{n}}=$ Regression coefficients

$B_{0}=$ Value of $\mu$ when independent variables are zero or the intercept on y-axis

For each subsoil, the dependent variables were liquid limit LL, and plastic limit PL. In addition, the independent variables were CEC, exchangeable cations and anions. The model was developed for each measured geotechnical parameter under the influence of each salt. For instance, the predictive model obtaining LL of subsoil A contaminated with $\mathrm{NaCl}$ salt with time was developed as follows:

The independent variables used were time $\Delta \mathrm{t}, \Delta \mathrm{CEC}$, $\Delta \mathrm{Na}^{+}$and $\Delta \mathrm{Cl}$, which were defined as:

$\Delta \mathrm{t} \quad=\mathrm{T}_{\mathrm{t}}-\mathrm{T}_{0}$

Where:

$\mathrm{T}_{\mathrm{t}} \quad=$ Predictive time in days,

To = Initial time taken as zero.

$\Delta \mathrm{CEC}=\mathrm{CEC}_{\mathrm{t}}-\mathrm{CEC}_{0}$
Where:

$\mathrm{CEC}_{\mathrm{t}}=$ Expected CEC at predictive year in $\mathrm{cmol} / \mathrm{kg}$

$\mathrm{CEC}_{0}=\mathrm{CEC}$ of uncontaminated soil in $\mathrm{cmol} / \mathrm{kg}$

$\Delta \mathrm{Na}^{+}=\mathrm{Na}^{+}{ }_{\mathrm{t}}-\mathrm{Na}^{+}{ }_{0}$

Where:

$\mathrm{Na}^{+} \mathrm{t}=$ Exchangeable $\mathrm{Na}^{+}$at predictive time in $\mathrm{cmol} /$ $\mathrm{kg}$

$\mathrm{Na}_{0}^{+}=$Exchangeable $\mathrm{Na}^{+}$for uncontaminated soil in $\mathrm{cmol} / \mathrm{kg}$

$\Delta \mathrm{Cl}-=\mathrm{Cl}_{\mathrm{t}}-\mathrm{Cl}_{0}$

Where:

$\mathrm{Cl}_{\mathrm{t}}$ = Exchangeable $\mathrm{Cl}-$ at predictive year in $\mathrm{cmol} /$ $\mathrm{kg}$

$\mathrm{Cl}_{0}^{-}=$Exchangeable $\mathrm{Cl}^{-}$for uncontaminated soil in $\mathrm{cmol} / \mathrm{kg}$

The dependent variable was liquid limit LL

On substituting parameters of Equations 2 to 5 into Equation 1, the general multiple regression equation for subsoil A liquid limit under the influence of $\mathrm{NaCl}$ at time $t$ reduces to

$\mathrm{LL}_{\mathrm{t}}=B_{0}+B_{1} \Delta \mathrm{t}+\mathrm{B}_{2} \Delta \mathrm{CEC}+B_{3} \Delta \mathrm{Na}^{+}+{ }_{B_{4}} \Delta \mathrm{Cl}$

Where:

$B_{0}=$ Liquid limit for uncontaminated soil

Regression coefficients estimation were obtained using Origin 8.0 statistical package [17]. The program input data were values of the four independent variables and one dependent variable. The output data among others were the regression coefficients and the $\mathrm{R}^{2}$ values. The $\mathrm{R}^{2}$ values revealed the level of prediction of dependent variable by independent ones.

The regression model obtained was shown in Equation 7:

$$
\begin{aligned}
\mathrm{LLt}= & 41.83+1.919 \times 10^{-3} \Delta \mathrm{t}-0.454 \Delta \mathrm{CEC}-0.304 \Delta \mathrm{Na}^{+} \\
& -2.38 \times 10^{-3} \Delta \mathrm{Cl}-
\end{aligned}
$$$$
\mathrm{R}^{2}=0.974(97.4 \%)
$$

The same procedure was repeated to generate the remaining predictive equations for subsoils $\mathrm{A}$ and $\mathrm{B}$ under the influence of $\mathrm{NaCl}, \mathrm{KNO}_{3}$ and $\mathrm{CaSO}_{4}$ salts. The symbols used are defined below

$\Delta \mathrm{K}^{+}=\mathrm{K}^{+}{ }_{\mathrm{t}}-\mathrm{K}^{+}{ }_{0}$

Where:

$\mathrm{K}^{+}{ }_{\mathrm{t}}=$ Exchangeable $\mathrm{K}^{+}$at predictive year in $\mathrm{cmol} / \mathrm{kg}$

$\mathrm{K}^{+}{ }_{0}=$ Exchangeable $\mathrm{K}^{+}$for uncontaminated soil in $\mathrm{cmol} / \mathrm{kg}$

$\Delta \mathrm{NO}_{3}=\mathrm{NO}_{3^{-} \mathrm{t}}-\mathrm{NO}_{30}{ }^{-}$

Where:

$\mathrm{NO}_{3 \mathrm{t}}=$ Exchangeable $\mathrm{NO}_{3}$ at predictive year in $\mathrm{cmol} / \mathrm{kg}$

$\mathrm{NO}_{30}=$ Exchangeable $\mathrm{NO}_{3}$ for uncontaminated soil in $\mathrm{cmol} / \mathrm{kg}$

$\Delta \mathrm{Ca}^{2+}=\mathrm{Ca}^{2+}{ }_{\mathrm{t}}-\mathrm{Ca}^{2+}{ }_{0}$ 
Where:

$\mathrm{Ca}^{2+}{ }_{\mathrm{t}}=$ Exchangeable $\mathrm{Ca}^{2+}$ at predictive time in $\mathrm{cmol} / \mathrm{kg}$

$\mathrm{Ca}^{2+}{ }_{0}=$ Exchangeable $\mathrm{Ca}^{2+}$ for uncontaminated soil in $\mathrm{cmol} / \mathrm{kg}$

$\Delta \mathrm{SO}_{4}^{2-}=\mathrm{SO}_{4}{ }^{2-} \mathrm{t}-\mathrm{SO}_{4}{ }^{2-} 0$

Where:

$\mathrm{SO}_{4}{ }^{2-} \mathrm{t}=$ Exchangeable $\mathrm{SO}_{4}{ }^{2-}$ at predictive time in $\mathrm{cmol} / \mathrm{kg}$

$\mathrm{SO}_{4}{ }^{2-0}=$ Exchangeable $\mathrm{SO}_{4}{ }^{2-}$ for uncontaminated soil in $\mathrm{cmol} / \mathrm{kg}$

The regression equations generated were shown in Results and Discussion section.

\section{Results and Discussion}

The grading curves of subsoils A and B are shown in Figures 2 and 3 . The result showed that the two subsoils were well-graded, but subsoil A was of more blended type than B. On the other hand, subsoil B has more particles less than $0.045 \mathrm{~mm}$ (29.17\%). In accordance with British Standard [18], both soils are clayey gravel (i.e. \% fines between 15 to $35 \%$ ).

The mean values of liquid limit of subsoils and contaminated soils were displayed in Tables 1 and 2 . Also, the average values of plastic limit measured in percentages were tabulated in Tables 3 and 4 . The chemical tests results for the two subsoils and contaminated soils measured in $\mathrm{cmol} / \mathrm{kg}$ were displayed in Tables 5 to 10. In Tables 5 to 10, the controlled soils tests results are included for easy comparison of the values obtained due to salts presence and when they are not. The multiple regression analyses led to the generation of equations for predicting future values of liquid and plastic limit (Tables 11 and 12).

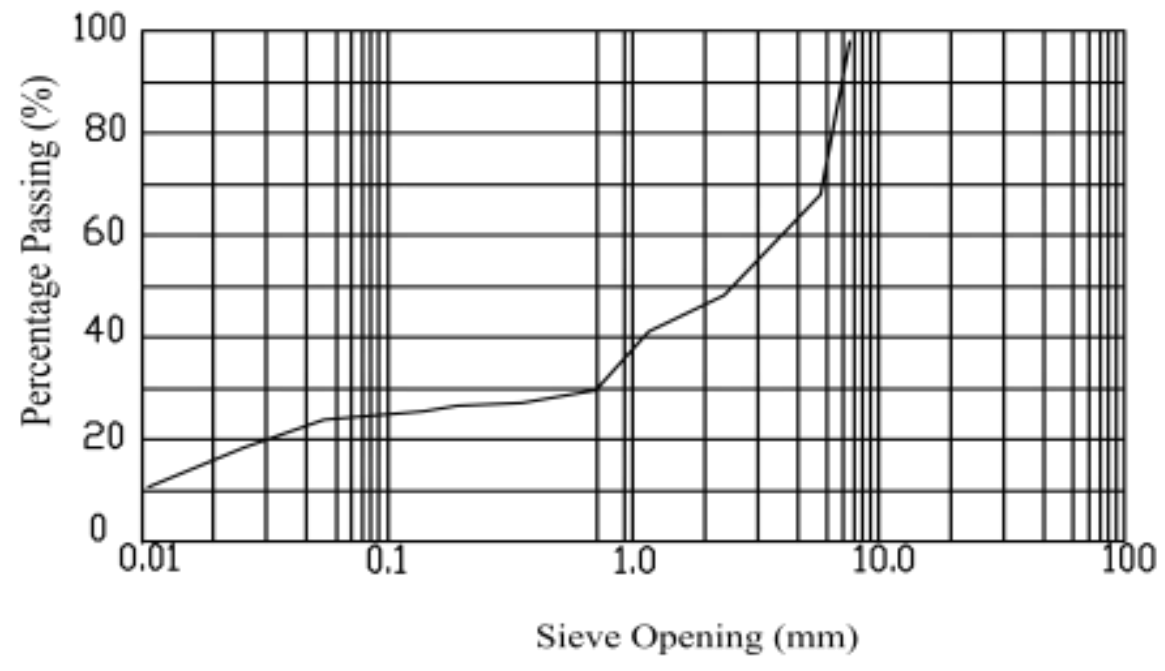

Figure 2. Grading Curve of Subsoil A

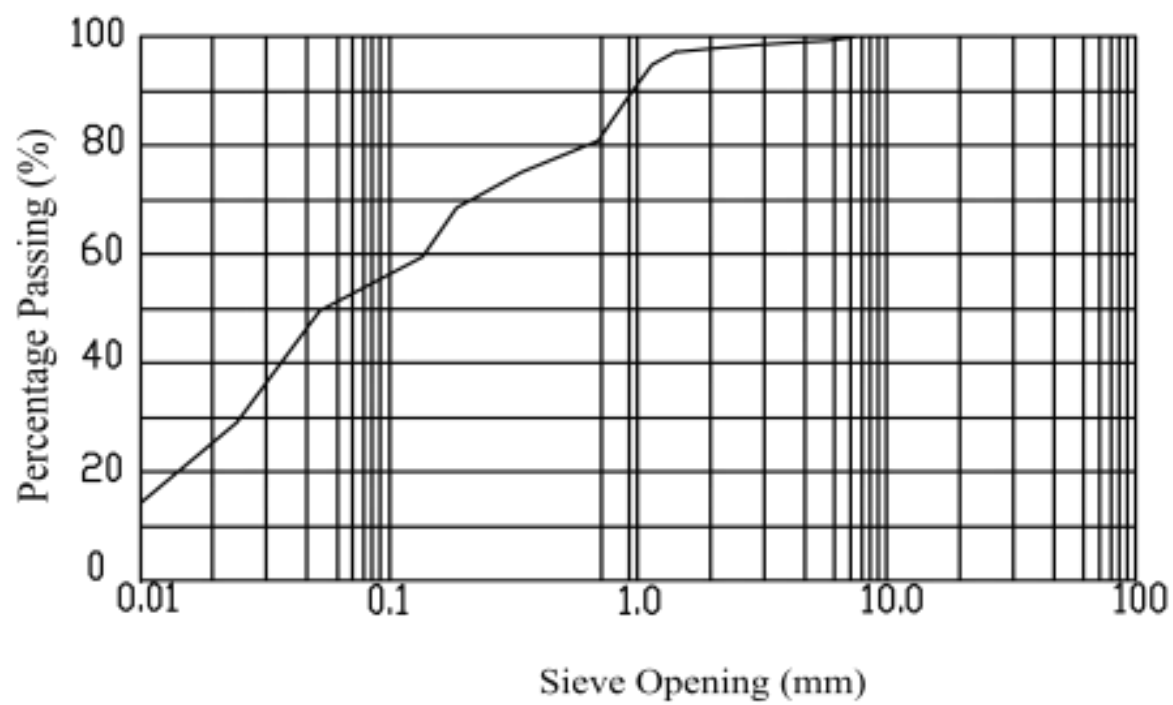

Figure 3. Grading Curve of Subsoil B 
Table 1. The Mean Values of Liquid Limit of Soil Samples of Subsoil A with Time

\begin{tabular}{|c|c|c|c|c|c|c|c|c|c|c|c|c|}
\hline \multirow{2}{*}{$\begin{array}{l}\text { Day of } \\
\text { soil test }\end{array}$} & \multicolumn{12}{|c|}{ Liquid limit (\%) } \\
\hline & ASC-10 & ASC-30 & ASC-50 & ASC-70 & AKN-10 & AKN-30 & AKN-50 & AKN-70 & ACS-10 & ACS-30 & ACS-50 & ACS-70 \\
\hline 0 & 42.00 & 42.00 & 42.00 & 42.00 & 42.00 & 42.00 & 42.00 & 42.00 & 42.00 & 42.00 & 42.00 & 42.00 \\
\hline 7 & 41.60 & 41.50 & 41.50 & 41.40 & 41.80 & 41.50 & 41.00 & 41.00 & 42.30 & 42.70 & 42.90 & 43.40 \\
\hline 14 & 41.40 & 41.30 & 41.20 & 41.20 & 41.40 & 41.30 & 40.80 & 40.50 & 42.60 & 42.90 & 43.30 & 43.80 \\
\hline 28 & 41.00 & 40.80 & 40.90 & 40.70 & 40.90 & 40.80 & 40.30 & 40.00 & 42.80 & 43.30 & 43.60 & 44.00 \\
\hline 56 & 40.00 & 40.30 & 40.10 & 39.60 & 40.50 & 40.30 & 39.80 & 39.60 & 43.10 & 43.50 & 43.80 & 44.20 \\
\hline 112 & 39.60 & 39.50 & 39.20 & 39.00 & 40.10 & 39.80 & 39.60 & 39.30 & 43.40 & 43.80 & 44.20 & 44.60 \\
\hline 196 & 39.40 & 39.30 & 38.80 & 38.70 & 39.90 & 39.50 & 39.40 & 39.20 & 43.70 & 44.20 & 44.50 & 44.90 \\
\hline 280 & 39.20 & 39.00 & 38.70 & 38.50 & 39.70 & 39.30 & 39.20 & 38.90 & 44.00 & 44.40 & 44.90 & 45.40 \\
\hline 364 & 39.10 & 38.80 & 38.50 & 38.40 & 39.60 & 39.20 & 39.00 & 38.70 & 44.20 & 44.50 & 45.00 & 45.50 \\
\hline
\end{tabular}

Table 2. The Average Values of Liquid Limit of Soil Samples B with Time

\begin{tabular}{ccccccccccccc}
\hline $\begin{array}{c}\text { Day of } \\
\text { soil test }\end{array}$ & BSC-10 & BSC-30 & BSC-50 & BSC-70 & BKN-10 & BKN-30 & BKN-50 & BKN-70 & BCS-10 & BCS-30 & BCS-50 & BCS-70 \\
\hline 0 & 36.00 & 36.00 & 36.00 & 36.00 & 36.00 & 36.00 & 36.00 & 36.00 & 36.00 & 36.00 & 36.00 & 36.00 \\
7 & 35.70 & 35.60 & 35.00 & 34.90 & 35.90 & 35.50 & 35.30 & 35.00 & 36.30 & 36.50 & 36.70 & 36.90 \\
14 & 35.50 & 35.30 & 34.80 & 34.50 & 35.70 & 35.30 & 34.80 & 34.50 & 36.60 & 36.90 & 37.10 & 37.30 \\
28 & 35.20 & 34.90 & 34.50 & 34.00 & 35.20 & 35.00 & 34.40 & 34.20 & 36.90 & 37.30 & 37.50 & 37.80 \\
56 & 34.80 & 34.50 & 34.00 & 33.40 & 34.80 & 34.60 & 34.00 & 33.70 & 37.20 & 37.60 & 37.90 & 38.10 \\
112 & 33.70 & 33.40 & 32.90 & 32.40 & 34.30 & 34.00 & 33.80 & 33.40 & 37.50 & 37.90 & 38.20 & 38.50 \\
196 & 33.20 & 32.80 & 32.30 & 31.90 & 33.70 & 33.60 & 33.50 & 33.00 & 38.00 & 38.20 & 38.50 & 38.80 \\
280 & 33.10 & 32.70 & 32.20 & 31.80 & 33.50 & 33.40 & 33.30 & 32.80 & 38.20 & 38.40 & 38.80 & 39.20 \\
364 & 33.00 & 32.60 & 32.10 & 31.70 & 33.40 & 33.30 & 33.10 & 32.70 & 38.30 & 38.50 & 38.90 & 39.30 \\
\hline
\end{tabular}

Table 3. The Mean Values of Plastic limit of Soil Samples A with Time

\begin{tabular}{|c|c|c|c|c|c|c|c|c|c|c|c|c|}
\hline \multirow{2}{*}{$\begin{array}{c}\text { Day of } \\
\text { soil test }\end{array}$} & \multicolumn{12}{|c|}{ Plastic limit (\%) } \\
\hline & ASC-10 & ASC-30 & ASC-50 & ASC-70 & AKN-10 & AKN-30 & AKN-50 & AKN-70 & ACS-10 & ACS-30 & ACS-50 & ACS-70 \\
\hline 0 & 19.60 & 19.60 & 19.60 & 19.60 & 19.60 & 19.60 & 19.60 & 19.60 & 19.60 & 19.60 & 19.60 & 19.60 \\
\hline 7 & 19.00 & 19.00 & 19.00 & 19.00 & 19.30 & 19. & 19.00 & 18.80 & 19.90 & 20.00 & 20.10 & 20.50 \\
\hline 14 & 18.70 & 18.90 & 18.80 & 18.70 & 19.00 & 18.80 & 18.50 & 18.30 & 20.30 & 20.50 & 20.60 & 20.80 \\
\hline 28 & 50 & 18 & 1 & 18 & 70 & 18 & 0 & 17 & 20.90 & 21.00 & 21 & 21.00 \\
\hline 56 & 30 & 17.90 & 17.60 & 17.10 & 18.30 & 18. & 17.80 & 17.40 & 21.00 & 21.20 & 21.40 & 21.30 \\
\hline 112 & 17.00 & 16.90 & 16.70 & 16.50 & 17.90 & 17.50 & 17.40 & 17.10 & 21.40 & 21.50 & 21.60 & 21.70 \\
\hline 196 & 16.60 & 16.40 & 16.30 & 16.20 & 17.50 & 17.30 & 17.10 & 16.90 & 21.60 & 22.00 & 22.30 & 22.50 \\
\hline 280 & 16.50 & 16.30 & 16.10 & 16.00 & 17.30 & 17.10 & 16.90 & 16.70 & 21.80 & 22.20 & 22.40 & 22.90 \\
\hline 364 & 16.40 & 16.20 & 16.00 & 15.80 & 17.20 & 17.00 & 16.80 & 16.60 & 21.90 & 22.40 & 22.50 & 23.00 \\
\hline
\end{tabular}

Table 4. The Average Plastic Limit Values of Soil Samples B with Time

\begin{tabular}{rcccccccccccc}
\hline \multirow{2}{*}{$\begin{array}{c}\text { Day of } \\
\text { soil test }\end{array}$} & BSC-10 & BSC-30 & BSC-50 & BSC-70 & BKN-10 & BKN-30 & BKN-50 & BKN-70 & BCS-10 & BCS-30 & BCS-50 & BCS-70 \\
\hline 0 & 22.00 & 22.00 & 22.00 & 22.00 & 22.00 & 22.00 & 22.00 & 22.00 & 22.00 & 22.00 & 22.00 & 22.00 \\
7 & 21.80 & 21.60 & 21.50 & 21.30 & 21.80 & 21.50 & 21.50 & 21.50 & 22.30 & 22.40 & 22.50 & 22.90 \\
14 & 21.60 & 21.20 & 21.30 & 21.00 & 21.40 & 21.30 & 21.00 & 20.80 & 22.70 & 22.90 & 22.90 & 23.20 \\
28 & 21.20 & 20.80 & 20.80 & 20.30 & 21.00 & 20.80 & 20.50 & 20.60 & 23.40 & 23.30 & 23.50 & 23.40 \\
56 & 20.70 & 20.30 & 20.00 & 19.50 & 20.50 & 20.50 & 20.00 & 20.20 & 23.60 & 23.60 & 23.90 & 23.70 \\
112 & 19.50 & 19.10 & 19.00 & 18.60 & 20.10 & 20.00 & 19.50 & 19.60 & 23.70 & 23.90 & 24.30 & 24.10 \\
196 & 18.90 & 18.80 & 18.30 & 18.30 & 19.80 & 19.50 & 19.30 & 19.20 & 24.40 & 24.40 & 24.70 & 24.50 \\
280 & 18.60 & 18.50 & 18.30 & 18.20 & 19.60 & 19.30 & 19.10 & 18.90 & 24.70 & 24.90 & 24.90 & 24.70 \\
364 & 18.50 & 18.40 & 28.20 & 18.10 & 19.50 & 19.20 & 19.00 & 18.70 & 24.90 & 25.30 & 25.00 & 24.80 \\
\hline
\end{tabular}

Table 5. The Average Chemical Properties of Soil Samples A Contaminated with $\mathrm{NaCl}$

\begin{tabular}{|c|c|c|c|c|c|c|c|c|c|c|c|c|}
\hline \multirow{3}{*}{$\begin{array}{l}\text { Day of } \\
\text { soil test }\end{array}$} & \multicolumn{12}{|c|}{ Chemical properties $(\mathrm{cmol} / \mathrm{kg})$} \\
\hline & \multicolumn{3}{|c|}{ ASC-10 } & \multicolumn{3}{|c|}{ ASC-30 } & \multicolumn{3}{|c|}{ ASC-50 } & \multicolumn{3}{|c|}{ ASC-70 } \\
\hline & $\mathrm{Na}$ & CEC & $\mathrm{Cl}$ & $\mathrm{Na}$ & CEC & $\mathrm{Cl}$ & $\mathrm{Na}$ & CEC & $\mathrm{Cl}$ & $\mathrm{Na}$ & CEC & $\mathrm{Cl}$ \\
\hline 0 & 0.10 & 1.57 & 21.80 & 0.10 & 1.57 & 21.80 & 0.10 & 1.57 & 21.80 & 0.10 & 1.57 & 21.80 \\
\hline 7 & 0.12 & 2.00 & 61.00 & 0.12 & 2.08 & 71.00 & 0.14 & 2.02 & 91.00 & 0.14 & 2.09 & 106.00 \\
\hline 14 & 0.18 & 2.20 & 106.00 & 0.18 & 2.32 & 118.00 & 0.21 & 2.48 & 120.00 & 0.24 & 2.48 & 121.00 \\
\hline 28 & 0.32 & 2.68 & 121.00 & 0.34 & 2.85 & 128.00 & 0.38 & 2.85 & 130.00 & 0.43 & 3.03 & 148.00 \\
\hline 56 & 0.54 & 3.14 & 185.00 & 0.61 & 3.54 & 206.00 & 0.68 & 3.97 & 245.00 & 0.74 & 4.11 & 391.00 \\
\hline 112 & 0.80 & 4.38 & 397.00 & 0.85 & 4.64 & 406.00 & 0.90 & 4.79 & 426.00 & 0.94 & 5.00 & 475.00 \\
\hline 196 & 1.24 & 5.33 & 473.00 & 1.40 & 5.71 & 483.00 & 1.58 & 6.06 & 490.00 & 1.63 & 5.96 & 494.00 \\
\hline 280 & 1.28 & 5.64 & 489.00 & 1.63 & 5.90 & 506.00 & 1.73 & 6.28 & 514.00 & 1.85 & 5.99 & 517.00 \\
\hline 364 & 1.30 & 5.83 & 497.00 & 1.72 & 5.90 & 519.00 & 1.81 & 6.40 & 520.00 & 1.92 & 6.02 & 528.00 \\
\hline
\end{tabular}


Table 6. The Average Chemical Properties of Soil Samples B Contaminated with $\mathrm{NaCl}$

\begin{tabular}{|c|c|c|c|c|c|c|c|c|c|c|c|c|}
\hline \multirow{3}{*}{$\begin{array}{c}\text { Day of } \\
\text { soil test }\end{array}$} & \multicolumn{12}{|c|}{ Chemical properties (cmol/kg) } \\
\hline & \multicolumn{3}{|c|}{ BSC-10 } & \multicolumn{3}{|c|}{ BSC-30 } & \multicolumn{3}{|c|}{ BSC-50 } & \multicolumn{3}{|c|}{ BSC-70 } \\
\hline & $\mathrm{Na}$ & $\mathrm{CEC}$ & $\mathrm{Cl}$ & $\mathrm{Na}$ & CEC & $\mathrm{Cl}$ & $\mathrm{Na}$ & CEC & $\mathrm{Cl}$ & $\mathrm{Na}$ & CEC & $\mathrm{Cl}$ \\
\hline 0 & 0.09 & 1.62 & 19.90 & 0.09 & 1.62 & 19.90 & 0.09 & 1.62 & 19.90 & 0.09 & 1.62 & 19.90 \\
\hline 7 & 0.11 & 2.10 & 34.10 & 0.12 & 2.14 & 68.30 & 0.13 & 2.16 & 79.00 & 0.14 & 2.18 & 104.00 \\
\hline 14 & 0.14 & 2.22 & 103.00 & 0.17 & 2.29 & 116.40 & 0.19 & 2.46 & 117.80 & 0.23 & 2.57 & 119.90 \\
\hline 28 & 0.30 & 2.65 & 110.90 & 0.32 & 2.79 & 126.10 & 0.36 & 2.86 & 128.10 & 0.43 & 3.02 & 146.10 \\
\hline 56 & 0.52 & 3.17 & 182.30 & 0.58 & 3.49 & 204.50 & 0.68 & 3.97 & 242.10 & 0.72 & 4.16 & 386.40 \\
\hline 112 & 0.78 & 4.44 & 395.20 & 0.80 & 4.64 & 403.60 & 0.86 & 4.80 & 423.80 & 0.91 & 5.02 & 472.50 \\
\hline 196 & 1.22 & 5.47 & 471.00 & 1.37 & 5.70 & 481.30 & 1.53 & 6.03 & 487.90 & 1.60 & 6.27 & 492.20 \\
\hline 280 & 1.42 & 5.67 & 489.50 & 1.56 & 5.87 & 493.70 & 1.64 & 6.79 & 499.10 & 1.95 & 6.87 & 513.50 \\
\hline 364 & 1.50 & 5.73 & 499.80 & 1.64 & 5.90 & 501.00 & 1.69 & 6.93 & 508.30 & 2.03 & 6.99 & 520.00 \\
\hline
\end{tabular}

Table 7. The Average Chemical Properties of Soil Samples A Contaminated with $\mathrm{KNO}_{3}$

\begin{tabular}{|c|c|c|c|c|c|c|c|c|c|c|c|c|}
\hline \multirow{3}{*}{$\begin{array}{c}\text { Day of } \\
\text { soil test }\end{array}$} & \multicolumn{12}{|c|}{ Chemical properties $(\mathrm{cmol} / \mathrm{kg}$ ) } \\
\hline & \multicolumn{3}{|c|}{ AKN-10 } & \multicolumn{3}{|c|}{ AKN-30 } & \multicolumn{3}{|c|}{ AKN-50 } & \multicolumn{3}{|c|}{ AKN-70 } \\
\hline & $\mathrm{K}$ & CEC & $\mathrm{NO}_{3}$ & K & CEC & $\mathrm{NO}_{3}$ & $\mathrm{~K}$ & CEC & $\mathrm{NO}_{3}$ & $\mathrm{~K}$ & CEC & $\mathrm{NO}_{3}$ \\
\hline 0 & 0.06 & 1.57 & 0.01 & 0.06 & 1.57 & 0.01 & 0.06 & 1.57 & 0.01 & 0.06 & 1.57 & 0.01 \\
\hline 7 & 0.38 & 1.83 & 0.01 & 0.41 & 1.99 & 0.01 & 0.45 & 2.19 & 0.01 & 0.49 & 2.26 & 0.02 \\
\hline 14 & 0.60 & 2.34 & 0.02 & 0.65 & 2.51 & 0.02 & 0.70 & 3.01 & 0.02 & 0.75 & 3.32 & 0.03 \\
\hline 28 & 0.83 & 2.88 & 0.03 & 0.88 & 3.06 & 0.04 & 0.93 & 3.67 & 0.04 & 0.98 & 3.42 & 0.04 \\
\hline 56 & 1.07 & 3.50 & 0.05 & 1.12 & 3.67 & 0.05 & 1.24 & 3.76 & 0.05 & 1.26 & 4.05 & 0.05 \\
\hline 112 & 1.33 & 4.19 & 0.06 & 1.38 & 4.36 & 0.06 & 1.42 & 4.47 & 0.06 & 1.57 & 4.73 & 0.07 \\
\hline 196 & 1.63 & 4.88 & 0.07 & 1.65 & 5.05 & 0.07 & 1.68 & 5.18 & 0.08 & 1.71 & 5.44 & 0.08 \\
\hline 280 & 1.73 & 4.99 & 0.08 & 1.76 & 5.11 & 0.09 & 1.79 & 5.34 & 0.09 & 1.82 & 5.61 & 0.09 \\
\hline 364 & 1.80 & 5.11 & 0.08 & 1.82 & 5.15 & 0.09 & 1.87 & 5.45 & 0.10 & 1.93 & 5.69 & 0.10 \\
\hline
\end{tabular}

Table 8. The Average Chemical Properties of Soil Samples of B Contaminated with $\mathrm{KNO}_{3}$

\begin{tabular}{|c|c|c|c|c|c|c|c|c|c|c|c|c|}
\hline \multirow{3}{*}{$\begin{array}{l}\text { Day of } \\
\text { soil test }\end{array}$} & \multicolumn{12}{|c|}{ Chemical properties (cmol/kg) } \\
\hline & \multicolumn{3}{|c|}{ BKN-10 } & \multicolumn{3}{|c|}{ BKN-30 } & \multicolumn{3}{|c|}{ BKN-50 } & \multicolumn{3}{|c|}{ BKN-70 } \\
\hline & $\mathrm{K}$ & CEC & $\mathrm{NO}_{3}$ & $\mathrm{~K}$ & CEC & $\mathrm{NO}_{3}$ & $\mathrm{~K}$ & CEC & $\mathrm{NO}_{3}$ & $\mathrm{~K}$ & CEC & $\mathrm{NO}_{3}$ \\
\hline 0 & 0.07 & 1.62 & 0.01 & 0.07 & 1.62 & 0.01 & 0.07 & 1.62 & 0.01 & 0.07 & 1.62 & 0.01 \\
\hline 7 & 0.39 & 1.90 & 0.01 & 0.42 & 2.08 & 0.01 & 0.45 & 2.26 & 0.01 & 0.51 & 2.34 & 0.01 \\
\hline 14 & 0.61 & 2.41 & 0.02 & 0.66 & 2.58 & 0.02 & 0.71 & 3.09 & 0.02 & 0.76 & 3.37 & 0.03 \\
\hline 28 & 0.85 & 2.94 & 0.03 & 0.89 & 3.13 & 0.03 & 0.94 & 3.74 & 0.04 & 0.99 & 3.48 & 0.04 \\
\hline 56 & 1.08 & 3.56 & 0.04 & 1.13 & 3.73 & 0.05 & 1.26 & 3.98 & 0.05 & 1.28 & 4.13 & 0.05 \\
\hline 112 & 1.35 & 4.26 & 0.06 & 1.39 & 4.41 & 0.06 & 1.44 & 4.53 & 0.06 & 1.58 & 4.79 & 0.07 \\
\hline 196 & 1.65 & 5.01 & 0.07 & 1.67 & 5.14 & 0.07 & 1.69 & 5.24 & 0.07 & 1.73 & 5.50 & 0.08 \\
\hline 280 & 1.87 & 5.11 & 0.09 & 1.87 & 5.63 & 0.08 & 1.80 & 5.86 & 0.08 & 1.79 & 5.63 & 0.08 \\
\hline 364 & 1.91 & 5.12 & 0.09 & 1.95 & 5.71 & 0.08 & 1.92 & 5.96 & 0.08 & 1.83 & 6.70 & 0.09 \\
\hline
\end{tabular}

Table 9. The Average Chemical Properties of Soil Samples A Contaminated $\mathrm{CaSO}_{4}$ Salt Solution

\begin{tabular}{|c|c|c|c|c|c|c|c|c|c|c|c|c|}
\hline \multirow{3}{*}{$\begin{array}{c}\text { Day of } \\
\text { soil test }\end{array}$} & \multicolumn{12}{|c|}{ Chemical properties $(\mathrm{cmol} / \mathrm{kg}$ ) } \\
\hline & \multicolumn{3}{|c|}{ ACS-10 } & \multicolumn{3}{|c|}{ ACS-30 } & \multicolumn{3}{|c|}{ ACS-50 } & \multicolumn{3}{|c|}{ ACS-70 } \\
\hline & $\mathrm{Ca}$ & $\mathrm{CEC}$ & $\mathrm{SO}_{4}$ & $\mathrm{Ca}$ & $\mathrm{CEC}$ & $\mathrm{SO}_{4}$ & $\mathrm{Ca}$ & CEC & $\mathrm{SO}_{4}$ & $\mathrm{Ca}$ & $\mathrm{CEC}$ & $\mathrm{SO}_{4}$ \\
\hline 0 & 0.30 & 1.57 & 18.40 & 0.30 & 1.57 & 18.40 & 0.30 & 1.57 & 18.40 & 0.30 & 1.57 & 18.40 \\
\hline 7 & 0.44 & 1.83 & 20.40 & 0.48 & 1.93 & 20.40 & 0.49 & 2.02 & 23.50 & 0.51 & 2.20 & 25.60 \\
\hline 14 & 0.53 & 2.18 & 30.40 & 0.54 & 2.27 & 35.25 & 0.60 & 2.34 & 40.10 & 0.65 & 2.51 & 43.50 \\
\hline 28 & 0.69 & 2.61 & 47.80 & 0.70 & 2.65 & 48.80 & 0.75 & 2.66 & 50.40 & 0.78 & 2.72 & 60.70 \\
\hline 56 & 0.88 & 2.84 & 63.80 & 0.96 & 2.96 & 71.40 & 0.98 & 3.02 & 74.40 & 1.12 & 3.23 & 74.43 \\
\hline 112 & 1.28 & 3.42 & 81.60 & 1.40 & 3.56 & 83.40 & 1.48 & 3.72 & 85.60 & 1.52 & 3.81 & 85.75 \\
\hline 196 & 1.60 & 4.00 & 87.40 & 1.70 & 4.17 & 90.30 & 1.80 & 4.38 & 93.40 & 1.93 & 4.62 & 94.50 \\
\hline 280 & 1.83 & 4.15 & 89.34 & 1.86 & 4.24 & 90.50 & 1.96 & 4.54 & 95.00 & 1.98 & 4.83 & 96.06 \\
\hline 364 & 1.91 & 4.20 & 90.21 & 1.93 & 4.32 & 90.61 & 2.03 & 4.61 & 95.08 & 2.05 & 4.91 & 97.11 \\
\hline
\end{tabular}


Table 10. The Average Chemical Properties of Soil Samples of B Contaminated $\mathrm{CaSO}_{4}$

\begin{tabular}{|c|c|c|c|c|c|c|c|c|c|c|c|c|}
\hline \multirow{3}{*}{$\begin{array}{l}\text { Day of } \\
\text { soil test }\end{array}$} & \multicolumn{12}{|c|}{ Chemical properties (cmol/kg) } \\
\hline & \multicolumn{3}{|c|}{ BCS-10 } & \multicolumn{3}{|c|}{ BCS-30 } & \multicolumn{3}{|c|}{ BCS-50 } & \multicolumn{3}{|c|}{ BCS-70 } \\
\hline & $\mathrm{Ca}$ & CEC & $\mathrm{SO}_{4}$ & $\mathrm{Ca}$ & CEC & $\mathrm{SO}_{4}$ & $\mathrm{Ca}$ & CEC & $\mathrm{SO}_{4}$ & $\mathrm{Ca}$ & CEC & $\mathrm{SO}_{4}$ \\
\hline 0 & 0.35 & 1.62 & 16.45 & 0.35 & 1.62 & 16.45 & 0.35 & 1.62 & 16.45 & 0.35 & 1.62 & 16.45 \\
\hline 7 & 0.49 & 1.88 & 18.23 & 0.53 & 1.98 & 18.56 & 0.54 & 2.04 & 21.73 & 0.48 & 2.18 & 23.91 \\
\hline 14 & 0.58 & 2.23 & 28.23 & 0.59 & 2.31 & 33.71 & 0.64 & 2.37 & 38.26 & 0.68 & 2.53 & 41.84 \\
\hline 28 & 0.73 & 2.66 & 45.89 & 0.75 & 2.64 & 46.63 & 0.79 & 2.73 & 48.34 & 0.82 & 2.79 & 58.67 \\
\hline 56 & 0.92 & 3.45 & 62.04 & 1.00 & 3.00 & 70.00 & 1.02 & 3.06 & 73.31 & 1.16 & 3.27 & 73.38 \\
\hline 112 & 1.32 & 3.43 & 80.00 & 1.44 & 3.61 & 82.21 & 1.52 & 3.75 & 83.52 & 1.57 & 3.86 & 83.70 \\
\hline 196 & 1.63 & 4.02 & 85.65 & 1.74 & 4.19 & 88.82 & 1.83 & 4.39 & 92.08 & 1.96 & 4.63 & 93.00 \\
\hline 280 & 1.80 & 4.31 & 87.76 & 1.88 & 4.52 & 90.04 & 1.98 & 4.61 & 94.76 & 2.04 & 4.85 & 95.78 \\
\hline 364 & 1.87 & 4.45 & 88.04 & 1.93 & 4.67 & 91.01 & 2.12 & 4.78 & 96.05 & 2.12 & 4.93 & 96.54 \\
\hline
\end{tabular}

Table 11. The Regression Models Equations for Burrow Pit Subsoil A

\begin{tabular}{clc}
\hline Salt & \multicolumn{1}{c}{ Model equation } & $\mathrm{R}^{2}$ \\
\hline $\mathrm{NaCl}$ & $\mathrm{LL}=42+1.919 \times 10^{-3} \Delta \mathrm{t}-0.454 \Delta \mathrm{CEC}+0.304 \Delta \mathrm{Na}^{+}-2.38 \times 10^{-3} \Delta \mathrm{Cl}-$ & 0.974 \\
& $\mathrm{PLt}=19.6+1.019 \times 10^{-3} \Delta \mathrm{t}-0.409 \Delta \mathrm{CEC}-4.54 \times 10^{-2} \Delta \mathrm{Na}^{+}-3.50 \times 10^{-3} \Delta \mathrm{Cl}-$ & 0.991 \\
$\mathrm{KNO}_{3}$ & $\mathrm{LL}=42+2.476 \times 10^{-3} \Delta \mathrm{t}-0.44 \Delta \mathrm{CEC}-0.302 \Delta \mathrm{K}^{+}-15.575 \Delta \mathrm{NO}_{3}-$ & 0.961 \\
& $\mathrm{PL}=19.6+1.423 \times 10^{-3} \Delta \mathrm{t}-0.456 \Delta \mathrm{CEC}-0.336 \Delta \mathrm{K}^{+}-8.829 \Delta \mathrm{NO}_{3}^{-}$ & 0.981 \\
$\mathrm{CaSO}_{4}$ & $\mathrm{LL}_{\mathrm{t}}=42-4.64 \times 10^{-4} \Delta \mathrm{t}+2.276 \Delta \mathrm{CEC}-2.059 \Delta \mathrm{Ca}^{2+}-7.12 \times 10^{-3} \Delta \mathrm{SO}_{4}{ }^{2-}$ & 0.908 \\
& $\mathrm{PL}_{\mathrm{t}}=19.6+1.624 \times 10^{-3} \Delta \mathrm{t}+1.874 \Delta \mathrm{CEC}-2.206 \Delta \mathrm{Ca}^{2+}+4.958 \times 10^{-3} \Delta \mathrm{SO}_{4}{ }^{2-}$ & 0.990 \\
\hline
\end{tabular}

Table 12. The Regression Models Equations for Burrow Pit Subsoil B

\begin{tabular}{clc}
\hline Salt & \multicolumn{1}{c}{ Model equation } & $\mathrm{R}^{2}$ \\
\hline $\mathrm{NaCl}$ & $\mathrm{LL}_{\mathrm{t}}=36+3.495 \times 10^{-3} \Delta \mathrm{t}-0.787 \Delta \mathrm{CEC}-8.06 \times 10^{-2} \Delta \mathrm{Na}^{+}-1.46 \times 10^{-3} \Delta \mathrm{Cl}-$ & 0.959 \\
& $\mathrm{PL}_{\mathrm{t}}=22+8.55 \times 10^{-4} \Delta \mathrm{t}-0.453 \Delta \mathrm{CEC}+0.204 \Delta \mathrm{Na}^{+}-4.20 \times 10^{-3} \Delta \mathrm{Cl}$ & 0.987 \\
$\mathrm{KNO}_{3}$ & $\mathrm{LL}_{\mathrm{t}}=36+2.205 \times 10^{-3} \Delta \mathrm{t}-0.619 \Delta \mathrm{CEC}-0.130 \Delta \mathrm{K}^{+}-8.162 \Delta \mathrm{NO}_{3}{ }^{-}$ & 0.960 \\
& $\mathrm{PL}_{\mathrm{t}}=22+1.989 \times 10^{-3} \Delta \mathrm{t}-0.509 \Delta \mathrm{CEC}-0.159 \Delta \mathrm{K}^{+}-12.715 \Delta \mathrm{NO}_{3}{ }^{-}$ & 0.990 \\
$\mathrm{CaSO}_{4}$ & $\mathrm{LL}_{\mathrm{t}}=36-2.09 \times 10^{-3} \Delta \mathrm{t}+0.746 \Delta \mathrm{CEC}+0.30 \Delta \mathrm{Ca}^{2+}+5.424 \times 10^{-3} \Delta \mathrm{SO}_{4}{ }^{2-}$ & 0.935 \\
& $\mathrm{PL}_{\mathrm{t}}=22+2.12 \times 10^{-3} \Delta \mathrm{t}+0.867 \Delta \mathrm{CEC}-1.193 \Delta \mathrm{Ca}^{2+}+1.761 \times 10^{-2} \Delta \mathrm{SO}_{4}{ }^{2-}$ & 0.972 \\
\hline
\end{tabular}

Table 13. Six Years Projection of Soils Liquid Limit at Different Levels of $\mathrm{Na}$ and $\mathrm{Cl}$

\begin{tabular}{|c|c|c|c|c|c|c|c|c|c|c|}
\hline \multirow{2}{*}{ Soil } & \multicolumn{3}{|c|}{ Chemical properties } & \multicolumn{7}{|c|}{ Predictive period (year) } \\
\hline & $\mathrm{Na}$ & CEC & $\mathrm{Cl}$ & 0.50 & 1.00 & 2.00 & 3.00 & 4.00 & 5.00 & 6.00 \\
\hline \multirow{3}{*}{ A } & 1.63 & 5.96 & 494.00 & 39.70 & 40.05 & 40.75 & 41.45 & 42.15 & 42.85 & 43.55 \\
\hline & 1.85 & 5.99 & 517.00 & 39.70 & 40.05 & 40.75 & 41.45 & 42.15 & 42.85 & 43.55 \\
\hline & 1.92 & 6.02 & 528.00 & 39.68 & 40.03 & 40.73 & 41.43 & 42.13 & 42.83 & 43.53 \\
\hline \multirow{3}{*}{ B } & 1.60 & 6.27 & 492.20 & 32.16 & 32.80 & 34.07 & 35.35 & 36.63 & 37.90 & 39.18 \\
\hline & 1.95 & 6.87 & 513.50 & 31.63 & 32.27 & 33.54 & 34.82 & 36.09 & 37.37 & 38.64 \\
\hline & 2.03 & 6.99 & 520.00 & 31.52 & 32.16 & 33.44 & 34.71 & 35.99 & 37.26 & 38.54 \\
\hline
\end{tabular}

Two consistency limits were considered, liquid and plastic limits. Both limits are used internationally for soil classification and strength correlations. The liquid limit was carried out for the two subsoils A and B (uncontaminated samples) and also for other soil samples (contaminated soils). The behaviour of soil samples contaminated with $\mathrm{NaCl}$ solution was different from those contaminated with $\mathrm{KNO}_{3}$ salt solution, and $\mathrm{CaSO}_{4}$. Tables 1 and 2 revealed the pattern of liquid limit of soil samples ASC-10 to ACS-70 and BSC-10 to BCS-70 under the influence of different concentrations of $\mathrm{NaCl}, \mathrm{KNO}_{3}$, and $\mathrm{CaSO}_{4}$ salts. The increase in $\mathrm{NaCl}$ salt concentration brought about $11.9 \%$ and $19.4 \%$ reduction in soil liquid and plastic limit respectively. Likewise, reduction in both liquid and plastic limits of $9.2 \%$ and $15.3 \%$ respectively were observed as a result of increase in $\mathrm{KNO}_{3}$ concentration with time. These findings were in agreement with those of references $[7,8,10,15]$. On the other hand, increase $\mathrm{CaSO}_{4}$ salt concentration led to increase in both liquid and plastic limits of order $9.2 \%$ and $17.3 \%$ respectively. The reduction rate and also increment rate in liquid and plastic limits were rapid initially and later dropped.

Tables 7 to 10 revealed that there were variations in the levels of exchangeable cations, anion and CEC of contaminated soil in each container with time. The $\mathrm{R}^{2}$ values of the set of equations obtained from the multiple regression analyses (Tables 11 and 12) were in the range of 0.908 to 0.990 . Based on the high values of $R^{2}$, the equations were used to investigate the effects of having fixed values of CEC, 
Table 14. Six Years Projection of Soils Plastic Limit at Different Levels of $\mathrm{Na}$ and $\mathrm{Cl}$

\begin{tabular}{|c|c|c|c|c|c|c|c|c|c|c|}
\hline \multirow{2}{*}{ Soil } & \multicolumn{3}{|c|}{ Chemical properties } & \multicolumn{7}{|c|}{ Predictive period (year) } \\
\hline & $\mathrm{Na}$ & CEC & $\mathrm{Cl}$ & 0.50 & 1.00 & 2.00 & 3.00 & 4.00 & 5.00 & 6.00 \\
\hline \multirow{3}{*}{$\mathrm{A}$} & 1.63 & 5.96 & 494.00 & 16.27 & 16.46 & 16.83 & 17.20 & 17.57 & 17.94 & 18.32 \\
\hline & 1.85 & 5.99 & 517.00 & 16.16 & 16.35 & 16.72 & 17.10 & 17.47 & 17.84 & 18.21 \\
\hline & 1.92 & 6.02 & 528.00 & 16.11 & 16.30 & 16.67 & 17.04 & 17.41 & 17.78 & 18.16 \\
\hline \multirow{3}{*}{ B } & 1.60 & 6.27 & 492.20 & 18.37 & 18.53 & 18.84 & 19.15 & 19.46 & 19.77 & 20.09 \\
\hline & 1.95 & 6.87 & 513.50 & 18.08 & 18.24 & 18.55 & 18.86 & 19.17 & 19.48 & 19.80 \\
\hline & 2.03 & 6.99 & 520.00 & 18.02 & 18.17 & 18.49 & 18.80 & 19.11 & 19.42 & 19.73 \\
\hline
\end{tabular}

Table 15. Six Years Projection of Soils Liquid Limit at Different Levels of $\mathrm{K}$ and $\mathrm{NO}_{3}$

\begin{tabular}{|c|c|c|c|c|c|c|c|c|c|c|}
\hline \multirow{2}{*}{ Soil } & \multicolumn{3}{|c|}{ Chemical properties } & \multicolumn{7}{|c|}{ Predictive period (year) } \\
\hline & $\mathrm{K}$ & $\mathrm{CEC}$ & $\mathrm{NO}_{3}$ & 0.50 & 1.00 & 2.00 & 3.00 & 4.00 & 5.00 & 6.00 \\
\hline \multirow{3}{*}{$\mathrm{A}$} & 1.71 & 5.44 & 0.08 & 39.13 & 39.58 & 40.48 & 41.38 & 42.28 & 43.18 & 44.09 \\
\hline & 1.82 & 5.61 & 0.09 & 38.83 & 39.28 & 40.19 & 41.09 & 41.99 & 42.89 & 43.79 \\
\hline & 1.93 & 5.69 & 0.10 & 38.66 & 39.11 & 40.01 & 40.91 & 41.81 & 42.71 & 43.61 \\
\hline \multirow{3}{*}{$\mathrm{B}$} & 1.73 & 5.50 & 0.08 & 33.20 & 33.60 & 34.41 & 35.21 & 36.02 & 36.82 & 37.63 \\
\hline & 1.79 & 5.63 & 0.08 & 33.06 & 33.47 & 34.27 & 35.08 & 35.88 & 36.69 & 37.49 \\
\hline & 1.83 & 6.70 & 0.09 & 32.37 & 32.77 & 33.58 & 34.38 & 35.19 & 35.99 & 36.80 \\
\hline
\end{tabular}

Table 16. Six years Projection of Soils Plastic Limit at Different Levels of $\mathrm{K}$ and $\mathrm{NO}_{3}$

\begin{tabular}{|c|c|c|c|c|c|c|c|c|c|c|}
\hline \multirow{2}{*}{ Soil } & \multicolumn{3}{|c|}{ Chemical properties } & \multicolumn{7}{|c|}{ Predictive period (year) } \\
\hline & $\mathrm{K}$ & $\mathrm{CEC}$ & $\mathrm{NO}_{3}$ & 0.50 & 1.00 & 2.00 & 3.00 & 4.00 & 5.00 & 6.00 \\
\hline \multirow{3}{*}{$\mathrm{A}$} & 1.71 & 5.44 & 0.08 & 16.90 & 17.16 & 17.68 & 18.20 & 18.72 & 19.24 & 19.76 \\
\hline & 1.82 & 5.61 & 0.09 & 16.68 & 16.94 & 17.46 & 17.98 & 18.50 & 19.02 & 19.54 \\
\hline & 1.93 & 5.69 & 0.10 & 16.56 & 16.81 & 17.33 & 17.84 & 18.37 & 18.89 & 19.41 \\
\hline \multirow{3}{*}{$\mathrm{B}$} & 1.73 & 5.50 & 0.08 & 19.22 & 19.58 & 20.31 & 21.03 & 21.76 & 22.48 & 23.21 \\
\hline & 1.79 & 5.63 & 0.08 & 19.07 & 19.43 & 20.15 & 20.88 & 21.61 & 22.33 & 23.06 \\
\hline & 1.83 & 6.70 & 0.09 & 18.48 & 18.84 & 19.57 & 20.29 & 21.02 & 21.74 & 22.47 \\
\hline
\end{tabular}

Table 17. Six Years Projection of Soils Liquid Limit at Different Levels of Ca and $\mathrm{SO}_{4}$

\begin{tabular}{|c|c|c|c|c|c|c|c|c|c|c|}
\hline \multirow{2}{*}{ Soil } & \multicolumn{3}{|c|}{ Chemical properties } & \multicolumn{7}{|c|}{ Predictive period (year) } \\
\hline & $\mathrm{Ca}$ & CEC & $\mathrm{SO}_{4}$ & 0.50 & 1.00 & 2.00 & 3.00 & 4.00 & 5.00 & 6.00 \\
\hline \multirow{3}{*}{$\mathrm{A}$} & 1.93 & 4.62 & 94.50 & 44.96 & 44.88 & 44.71 & 44.54 & 44.37 & 44.20 & 44.03 \\
\hline & 1.98 & 4.83 & 96.06 & 45.33 & 45.25 & 45.08 & 44.91 & 44.74 & 44.57 & 44.40 \\
\hline & 2.05 & 4.91 & 97.11 & 45.36 & 45.28 & 45.11 & 44.94 & 44.77 & 44.60 & 44.43 \\
\hline \multirow{3}{*}{ B } & 1.96 & 4.63 & 93.00 & 38.77 & 38.38 & 37.62 & 36.86 & 36.10 & 35.33 & 34.57 \\
\hline & 2.04 & 4.85 & 95.78 & 38.97 & 38.59 & 37.83 & 37.07 & 36.30 & 35.54 & 34.78 \\
\hline & 2.12 & 4.93 & 96.54 & 39.06 & 38.68 & 37.91 & 37.15 & 36.39 & 35.63 & 34.86 \\
\hline
\end{tabular}

Table 18. Six Years Projection of Soils Plastic Limit at Different Levels of Ca and $\mathrm{SO}_{4}$

\begin{tabular}{ccccccccccc}
\hline \multirow{2}{*}{ Soil } & \multicolumn{3}{c}{ Chemical properties } & \multicolumn{7}{c}{ Predictive period (year) } \\
\cline { 2 - 26 } & $\mathrm{Ca}$ & $\mathrm{CEC}$ & $\mathrm{SO}_{4}$ & 0.50 & 1.00 & 2.00 & 3.00 & 4.00 & 5.00 & 6.00 \\
\hline \multirow{3}{*}{$\mathrm{A}$} & 1.93 & 4.62 & 94.50 & 22.40 & 22.69 & 23.29 & 23.88 & 24.47 & 25.06 & 25.66 \\
& 1.98 & 4.83 & 96.06 & 22.69 & 22.99 & 23.58 & 24.17 & 24.77 & 25.36 & 25.95 \\
& 2.05 & 4.91 & 97.11 & 22.69 & 22.99 & 23.58 & 24.17 & 24.77 & 25.36 & 25.95 \\
& 1.96 & 4.63 & 93.00 & 24.39 & 24.78 & 25.55 & 26.33 & 27.11 & 27.88 & 28.65 \\
B & 2.04 & 4.85 & 95.78 & 24.54 & 24.93 & 25.70 & 26.48 & 27.25 & 28.02 & 28.80 \\
& 2.12 & 4.93 & 96.54 & 24.53 & 24.91 & 25.69 & 26.46 & 27.24 & 28.01 & 28.78 \\
\hline
\end{tabular}

exchangeable cation and exchangeable anion in the subsoils for a period of six years. Table 13 revealed that at constant levels of $\mathrm{Na}, \mathrm{Cl}$, and $\mathrm{CEC}$, liquid limit values of the two subsoils reached their uncontaminated state values at fourth year of contamination. In addition, further increments were recorded beyond fourth year of salt soil interaction. On the other hand, the values of plastic limit subsoils A and B (Table 14) were approaching their uncontaminated values at sixth years of contamination.

Similar trends were observed when levels of $\mathrm{K}, \mathrm{NO}_{3}$, and CEC in subsoils A and B were kept constant for about six years as shown in Tables 15 and 16. At constant levels of $\mathrm{Ca}, \mathrm{SO}_{4}$, and CEC (Table 17), 
reduction in subsoil A liquid limit values were observed and their values at sixth year of contamination were approaching its uncontaminated value. Subsoil B uncontaminated liquid limit value was attained around fourth year of contamination. On the other hand, subsoil A and B plastic limit values increased with time and their uncontaminated values were never attained within the sixth year of projection (Table 18). Subsoils A and B contaminated with $\mathrm{NaCl}$, and $\mathrm{KNO}_{3}$ separately could regain their initial or original state plastic and liquid limits values with time. In contrast, subsoil contaminated with $\mathrm{CaSO}_{4}$ could regain its uncontaminated liquid limit value with time but not the plastic limit.

The best approach to explain the above finding is to apply the principle of ion exchange theory among soil clay minerals. During the ions exchange, the bond created by $\mathrm{Ca}^{2+}$ divalent ion was stronger than those of $\mathrm{Na}^{+}$and $\mathrm{K}^{+}$monovalent ions. In order for this bond to be weakened, more water molecule would be needed. In case of monovalent ion like $\mathrm{Na}^{+}$, twice $\mathrm{Na}^{+}$ ion would be needed to replace a divalent cation such as $\mathrm{Ca}^{2+}$. Therefore, the bond created would not be as strong as that of divalent type. As more monovalent ions are present, the bond would continue to be weakened and will require less water to break. Hence, $\mathrm{Ca}^{2+}$ dominated subsoils would have higher liquid and plastic limits than those of $\mathrm{Na}^{+}$and $\mathrm{K}^{+}$ types. Also, since $\mathrm{K}^{+}$is higher than $\mathrm{Na}^{+}$in ion exchange trend, the bond formed with $\mathrm{K}^{+}$would be stronger than that formed by $\mathrm{Na}^{+}$. Consequently, reduction in liquid and plastic limits rate would be higher in $\mathrm{Na}^{+}$dominated soil than that of $\mathrm{K}^{+}$soils.

\section{Conclusion}

The presence of $\mathrm{NaCl}$ and $\mathrm{KNO}_{3}$ in subsoils brought about reduction in both liquid and plastic limits at the initial age of contamination. On the contrary, $\mathrm{CaSO}_{4}$ presence resulted to initial increase in liquid limit. There is high tendency for the soil to regain its original uncontaminated limits. The electrostatic bond created by $\mathrm{Ca}^{2+}$ ion and the weakening of bond due to replacement with monovalent ions are not permanent. Therefore, the age of salt contamination is very important in order to know the contaminated soil liquid and plastic limits.

\section{References}

1. Whitlow, R., Basic Soil Mechanics, first edition, Longman, Malaysia, 1997.

2. Valsaraj, K.T., Elements of Environmental Engineering-Thermodynamics and Kinetics, first edition, Lewis Pub., New York, 2000.

3. Fakayode, S.O. and Olu-Owolabi, B.I., Heavy Metal Contamination of Roadside Top Soil in Osogbo, Nigeria; Its Relationship to Traffic Density and Proximity to Highways, Journal of
Environmental Geology, Springer Verlag, 44(2), 2003, pp. 150-157.

4. Gulec, N., Gunal, B., and Erler, A., Assessment of Soil and Water Contamination Around an Ash-disposal Site; A Case Study from the Seuitomer Coal-fired Power Plant in Western Turkey, Journal of Environmental Geology, Springer Verlag, 40(3), 2000, pp. 331-344.

5. Govil, P., Reddy, G., and Krishna, A., Contamination of Soil due to Heavy Metals in the Patancheru Industrial Development Area, Andhra, India, Journal of Environmental Geology, Springer Verlag, 41(3 and 4), 2000, pp. 461-469.

6. Barbour, S.L. and Fredlund, D.G., Mechanisms of Osmotic Flow and Volume Change in Clay Soils, Canadian Geotechnical Engineering Journal, NRC Canada, 26, 1989, pp. 551-562.

7. Maio, C.D., Exposure of Bentonite to Salt Solution Osmotic and Mechanical Effects, Geotechnique, Thomas Telford Ltd, 46, 1996, pp. 695- 707.

8. Schmitz, R.M. and Van Paasen, L.A., The Decay of Liquid Limit of Clays with Increasing Salt Concentration, Lingeokring Newsletter, 9(1), 2003, pp. 10-14.

9. Ohtsubo, M., Egashirak, K., Koumoto, T., and Bergado, D.T., Mineralogy and Chemistry and their Correlation with the Geotechnical Index Properties of Bangkok Clay Comparison with Ariake Clay, Journal of Japanese Geotechnical Society, Tokyo, 40(1), 2000, pp. 11-21.

10. Torrance, J.K. and Ohtsubo, M., Ariake Bay Quick Clay a Comparison with the General Model, Journal of Soils and Foundation, Japanese Geotechnical Society, Tokyo, 35(1), 1995, pp. 11-19.

11. Gleason, M.H., Daniel, D.E., and Eykholt, G.R., Calcium and Sodium Bentonite for Hydraulic Containment Applications, Journal of Geotechnical and Geoenvironmental Engineering, ASCE, 123, 1997, pp. 438-445.

12. Lee, J.M., Shackelford, C.D., Benson, C.H., Jo, H.Y., and Edil, T.B., Correlating Index Properties and Hydraulic Conductivity of Geosynthetic Clay Liners, Journal of Geotechnical and Geoenvironmental Engineering, ASCE, 131, 2005, pp. 1319-1329.

13. Park, J., Vipulanandan, C., Kim, J.W., and Oh, M.H., Effects of Surfactants and Electrolyte Solutions on the Properties of Soil, Journal of Environmental Geology, Springer Verlag, 49, 2006, pp. 977- 989.

14. Barbour, S.L. and Yang, N., A Review of the Interaction of Clay-brine Interactions on the Geotechnical Properties of Montmorillonitic Clayey Soils from Canada, Canadian Geotechnical Engineering Journal, NRC Canada, 30, 1993, pp. 920 934. 
15. Arasan, S. and Yetimoglu, T., Effect of Inorganic Salt Solutions on the Consistency Limit of Two Clays, Turkish Journal of Engineering Environmental Science, 32, 2003, pp. 1-9.

16. BS 1377, Methods of London Test for Soils for Civil Engineering Purposes, 1991, BSI,
17. OriginLab, Origin 8.0: A New Multi-sheet Workbook to Facilitate the Management of Data, Images, Graphs and Results, www.originlab.com, 2007.

18. Larsen, P.V., Module 3-Multiple Linear Regression, Retrieved April 10, 2008 from http://statmaster.sdu.dk/courses/st111/module03/index.html, 2008. 